\title{
A database of high and medium-resolution stellar spectra ${ }^{\star, \star \star}$
}

\author{
Ph. Prugniel ${ }^{1}$ and C. Soubiran ${ }^{2}$ \\ 1 CRAL-Observatoire de Lyon, CNRS UMR 142, 69561 Saint-Genis Laval, France \\ 2 Observatoire de Bordeaux, CNRS UMR 5804, BP 89, 33270 Floirac, France
}

Received 27 November 2000 / Accepted 26 January 2001

\begin{abstract}
We present a database of 908 spectra of 709 stars obtained with the ELODIE spectrograph at the Observatoire de Haute-Provence. 52 orders of the echelle spectra have been carefully fitted together to provide continuous, high-resolution spectra in the wavelength range $\lambda \lambda=410-680 \mathrm{~nm}$. The archive provides a large

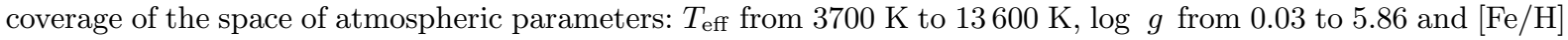
from -2.8 to +0.7 . At the nominal resolution, $R=42000$, the mean signal-to-noise ratio is 150 per pixel. The spectra given at this resolution are normalized to their pseudo-continuum and are intended to serve for abundance studies, spectral classification and tests of stellar atmosphere models. A lower resolution version of the archive, at $R=10000$, is calibrated in physical flux with a broad-band photometric precision of $2.5 \%$ and narrow-band precision of $0.5 \%$. It is well suited to stellar population synthesis of galaxies and clusters, and to kinematical investigations of stellar systems. The archive is distributed in FITS format through the HYPERCAT and CDS databases.
\end{abstract}

Key words. atlases - stars: abundances - stars: atmospheres - stars: fundamental parameters - galaxy: stellar content

\section{Introduction}

Spectral libraries covering the HR diagram with medium to high resolution and large spectral range are essential tools in astronomy. We have identified two areas where there will be a special need of such libraries in the coming years: the automated parameterization of stellar spectra and the spectral synthesis of stellar populations of galaxies. New multi-object spectrographs on large telescopes, such as Giraffe on the VLT, will soon make it possible to observe hundreds of objects during the same exposure with an unprecedented resolution and spectral coverage. The challenge will be to extract the maximal information in a reasonable time.

For stars, medium to high resolution spectra are the basis for determining radial velocities, atmospheric parameters $\left(T_{\text {eff }}, \log g,[\mathrm{Fe} / \mathrm{H}]\right)$ and eventually projected rotational velocities, $v \sin i$. A new method, called TGMET, is emerging to make the parameterization of stellar spec-

Send offprint requests to: Ph. Prugniel,

e-mail: prugniel@obs.univ-lyon1.fr

* Based on observations made on the $193 \mathrm{~cm}$ telescope at the Haute-Provence Observatory, France.

$\star \star$ Table 1 is only available in electronic form at the CDS via anonymous ftp to cdsarc.u-strasbg.fr (130.79.128.5)

or via

http://cdsweb.u-strasbg.fr/cgi-bin/qcat?J/A+A/369/1048 tra in terms of temperature, gravity and metallicity fast and automatic (Katz et al. 1998). It relies on the direct comparison of a target spectrum to a library of reference spectra with known atmospheric parameters (Soubiran et al. 1998). TGMET was originally dedicated to the measurement of F, G, K stars observed with ELODIE. The present paper continues the TGMET project. The two major points addressed are (1) the extension of the reference library to all spectroscopic types and luminosity classes and its densification, and (2) to resample the spectra in wavelength (i.e. remove the instrumental signature in ELODIE spectra and connect the echelle orders together) in order to enable comparison of spectra of any origin.

The TGMET project appeared to be also very useful for extragalactic studies where medium resolution libraries with large coverage in stellar parameters are needed. Stellar libraries, calibrated in flux, are used to model composite spectra through stellar population synthesis (e.g. the PEGASE program, Fioc \& Rocca-Volmerange 1997). An important issue is to disentangle the effects of age and metallicity and it has been demonstrated that the spectral resolution is a key factor (Worthey \& Ottviani 1997, hereafter WO97; Vazdekis 1999). To benefit from a resolution higher than about $R=2000$ both the internal kinematics and the characteristics of the stellar populations must 
be fitted simultaneously. Making available an extended library of stellar spectra is the first step in this direction.

Current libraries are based on low resolution spectroscopy (e.g. Jacoby et al. 1984; Serote Roos et al. 1996; Pickles 1998) or they are restricted to a limited area of the HR diagram (e.g. Montes et al. 2000 and Soubiran et al. 1998 for F, G, K stars). Here we present an homogeneous dataset of spectra which fills the deficiency of such libraries. The sample of stars and associated data are presented in Sect. 2. Sections 3 and 4 describe the two phases of the data processing. In Sect. 5 we evaluate the quality of the archive. The access to the data through HYPERCAT $^{1}$ or at the Centre de Données astronomiques de Strasbourg ${ }^{2}$ are described in Sect. 6.

\section{Stellar content and data}

\subsection{Physical parameters of the stars}

The present sample was assembled by merging the stellar library of F, G, K stars (Soubiran et al. 1998) serving as a basis for the TGMET program with additional spectra taken from other observing programs and from the near-to-line ELODIE archive (http://www.obs-hp.fr). The spectra of the database correspond to stars which have been selected because they have published values of $T_{\text {eff }}$ or $\left(T_{\text {eff }}, \log g,[\mathrm{Fe} / \mathrm{H}]\right)$, or reliable estimations of the absolute magnitude $M_{V}$, deduced from Hipparcos parallaxes. The main source of atmospheric parameters is the catalogue of $[\mathrm{Fe} / \mathrm{H}]$ determinations, 1996 and 2001 versions (Cayrel de Strobel et al. 1997 and 2001) but two other sources have been also used: Carney et al. (1994) and Thévenin (1998). Effective temperatures were also taken in the lists published by Blackwell \& Lynas-Gray (1998), Alonso et al. (1996a) and Alonso et al. (1999a), or calculated from the colour indices $V-K$ or $b-y$ following the relations $T_{\text {eff }}=f($ colour, $[\mathrm{Fe} / \mathrm{H}])$ established by Alonso et al. (1996b) and (1999b) for dwarfs and giants respectively. Each star had $\left(T_{\text {eff }}, \log g,[\mathrm{Fe} / \mathrm{H}]\right)$ estimated by averaging the determinations found in the literature, giving a half weight to old references and Strömgren photometry and a double weight to $T_{\text {eff }}$ determined by Blackwell \& Lynas-Gray (1998). As in the TGMET library, the parameters were then labelled according to their reliability which was estimated from the number of determinations and their standard deviation around the mean. The reliability scale runs from 0 to 4 , with the highest reliability 4 corresponding to uncertainties lower than $80 \mathrm{~K}$ in $T_{\text {eff }}$, and 0.06 dex in $[\mathrm{Fe} / \mathrm{H}]$. These uncertainties can reach $115 \mathrm{~K}$ and 0.09 dex for reliability 3 , and $150 \mathrm{~K}$ and 0.11 dex for the reliability 2 . Reliability 1 is attributed for parameters based on old determinations or photometry, or presenting large discrepancies between references. Reliability 0 means that no reference on atmospheric parameters was

\footnotetext{
1 http://www-obs.univ-lyon1.fr/hypercat/11/ spectrophotometry.html

2 http://cdsweb.u-strasbg.fr/cats/III.htx
}

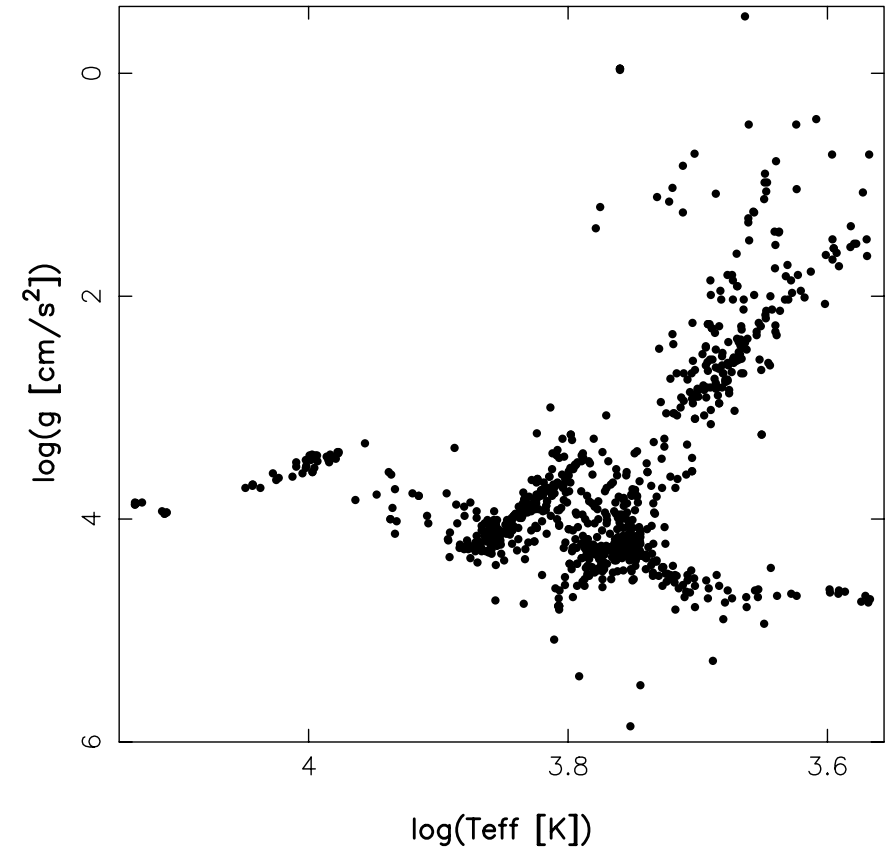

Fig. 1. $T_{\text {eff }}$ vs. $\log g$ for the 709 stars of the archive. For the sake of homogeneity, the atmospheric parameters presented here are not from the literature, which is incomplete, but were estimated with the current version of TGMET (Sect. 5.3.3)

found in the literature. The database consists of 908 spectra corresponding to 709 different stars which are shown in the plane $\left(\log T_{\text {eff }}, \log g\right)$ in Fig. 1 and in the plane $\left(\log T_{\text {eff }},[\mathrm{Fe} / \mathrm{H}]\right)$ in Fig. 2. $T_{\text {eff }}$ ranges from $3700 \mathrm{~K}$ to $13600 \mathrm{~K}, \log g$ ranges from 0.03 to 5.86 and $[\mathrm{Fe} / \mathrm{H}]$ ranges from -2.8 to +0.7 . In these two plots, new estimations of $\left(T_{\text {eff }}, \log g,[\mathrm{Fe} / \mathrm{H}]\right)$ were used instead of those from the literature, which are incomplete. These new estimations were obtained with the current version of TGMET which is still under development (a paper presenting this new version is in preparation and a description is given in the electronic version of the archive). Atmospheric parameters from the literature together with new estimations and measured line indices from the spectra presented here are given in Table 1, available only in electronic form (see Sect. 6).

More information on each star is available in the header of the FITS spectra. It includes the absolute magnitude $M_{V}$ from Hipparcos parallaxes when available. A scale of reliability was also established from the precision of the parallax and $V$ magnitude from Tycho-2 (Høg et al. 2000), 4 corresponding to parallaxes with a precision better than $10 \%$. Also available in the FITS headers are the spectral types, $(V, B-V)$ taken from the Tycho-2 catalogue (converted from the Tycho- $2 B_{\mathrm{T}}$ and $\left.V_{\mathrm{T}}\right)$ and radial velocities usually measured at the telescope. The on-line cross-correlation technique was used to measure the radial velocity of strong-lined spectra, corresponding to moderate effective temperatures up to $6500 \mathrm{~K}$ but for hotter stars, the radial velocity was estimated 


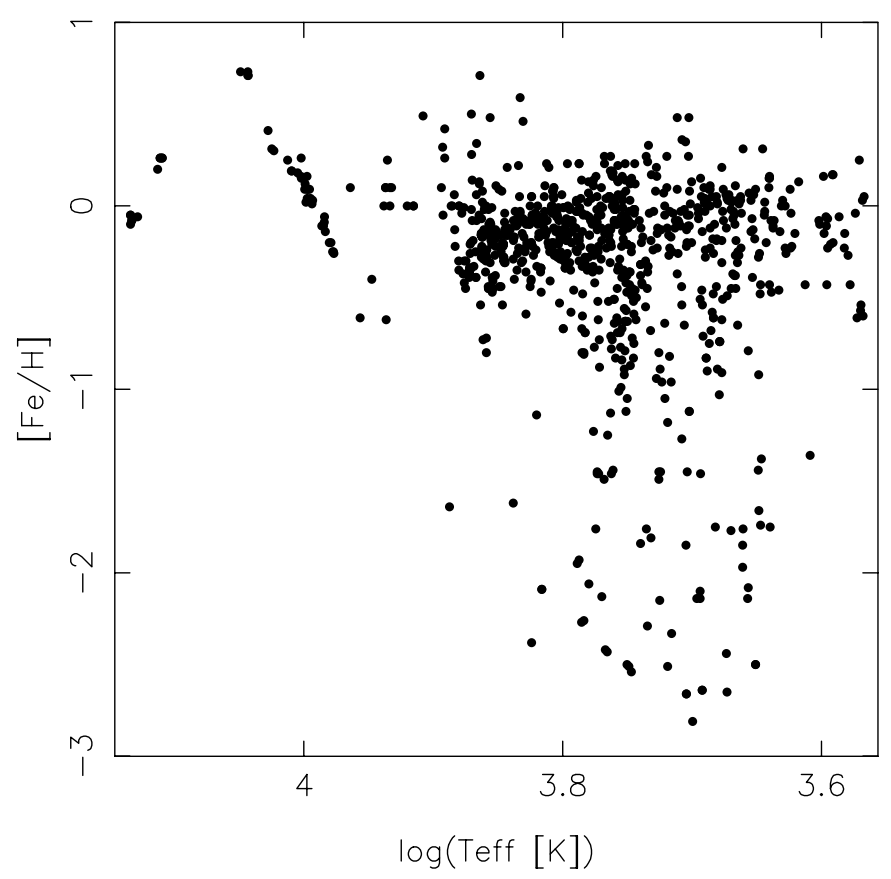

Fig. 2. Distribution of the 709 stars of the archive in the plane $T_{\text {eff }}$ vs. $[\mathrm{Fe} / \mathrm{H}]$ estimated with the current version of TGMET (Sect. 5.3.3)

using a least-squares deconvolution technique (Donati et al. 1997).

The database includes several stars with line profiles broadened by rotation, macroturbulence or binarity and a few stars with spectral peculiarities (Ap, Am, emission line stars...). These pecularities are indicated in the headers.

\subsection{ELODIE spectra}

ELODIE provides a spectral range of 390-680 $\mathrm{nm}$ recorded in a single exposure as 67 orders on a $1 \mathrm{~K}$ CCD at a mean resolving power of 42000 . Optimal extraction and wavelength calibration are automatically performed by the online reduction software TACOS. Complete information on ELODIE can be found in Baranne et al. (1996) and in the TACOS user manual by Queloz (1996). The global efficiency of the instrument drops in the blue by a factor 5 at $440 \mathrm{~nm}$ for a solar-type star and by a factor 50 at $390 \mathrm{~nm}$. Hence we decided to limit the spectral range to $\lambda \lambda=410-680 \mathrm{~nm}$.

The typical signal-to-noise $(S / N)$ ratio per pixel at $550 \mathrm{~nm}$ is 150 (it is higher than 80 for more than $90 \%$ of the spectra). Several spectra with a lower $S / N$, down to 35 , correspond generally to faint deficient stars which have been included in the database for a better sampling of the parameter space.

For the present purpose, the 908 spectra of the database were processed to provide $1 \mathrm{D}$ spectra rebinned in wavelength and calibrated in absolute fluxes. Since the main goal of the archive was to construct a library of spectra representative of the various stellar types and an interpolated grid of spectra covering the parameter space, all the spectra were reduced to the rest-frame. The archive was prepared from the flat-fielded and order-extracted TACOS spectra in two main steps. (1) The orders were deblazed, the spikes due to cosmic rays and telluric lines were masked, all the orders were connected in one spectrum, and the pseudo-continuum normalization was computed. (2) The spectra were calibrated into "physical" flux, i.e. above the atmosphere.

\section{Strengthening and normalization to the pseudo-continuum}

This phase of the data processing takes the TACOS spectra and delivers order-connected spectra with a constant wavelength step. Both the instrumental and continuum fluxes are evaluated.

The operations follow these steps:

- Strengthen the spectra by dividing them by a deblazing function;

- Compute the pseudo-continuum normalization and analyze the "shape" of each order to determine a correction to the diffuse-light subtraction;

- Mask spikes and telluric lines;

- Convolve and rebin with two desired resolutions $(R=$ 10000 and $R=42000$ ) to $1 \mathrm{D}$ spectra.

\subsection{De-blazing and pseudo-continuum normalization}

The instrument, hard- and soft-ware, was mostly designed as a "velocimeter" and hence the spectrophotometric quality was not optimized. The standard flat-field correction and order extraction was applied as described in Baranne et al. (1996). However, the procedure is not sufficient to connect the orders into a single spectrum, because the blaze function is imperfectly corrected. Actually, after the standard correction, each order presents a residual "curvature" of about $2 \%$. This effect is certainly due to diffuse light within the spectrograph which is imperfectly subtracted by the TACOS program, this is a common difficulty with echelle spectrographs.

To solve this problem we had to analyze this residual curvature on the actual spectra and correct it. Obviously, it is a risky approach since the spectrum itself is used to determine the correction. To minimize possible biases, the residual curvature is modeled with only a few parameters: each order is represented by a second degree polynomial whose coefficients are not independent from order to order, but are themselves second degree polynomials of the order number. The total effect of the diffuse light is modeled by only 3 coefficients.

Practically, we divide the TACOS spectrum by a deblazing function determined from a tungsten-lamp spectrum (internal flat-field) and corrected using 3 metaldeficient stars (because the blaze function appears to have slight systematic differences between internal flat-field and star). 
To analyze the "residual" curvature of each order, we normalized the spectrum to its pseudo-continuum, defined as a $3^{\text {rd }}$ degree spline interpolation from one point in each order. Each point is determined by fitting a second degree polynomial to the order weighted with a mask to avoid as much as possible the stellar lines. The fit is iterated after $\kappa-\sigma$ clipping and the remaining bias due to the weak stellar lines is statistically corrected. The latter correction is based on the analysis of the skewness of the distribution of residuals. The weighting mask is prepared from a previous iteration of the reduction of the whole archive: for each wavelength point it gives the probability of having a stellar line (we started with no weight).

Though the visual inspection of the strengthened spectra appears satisfactory, the (unsuccessful) search for possible biases is presented in Sect. 5 .

After the correction for diffuse light was applied, the pseudo-continuum was re-computed. These normalized spectra are scoped for abundance studies, and determination of the atmospheric parameters with TGMET.

All the information permitting calculation of the detected flux (in electrons) is kept in the form of a table extension attached to the FITS image. The $S / N$ of each pixel is also kept in a separate extension. The detailed description of the FITS files is given with the electronic version of the tables and spectra.

\subsection{Cosmic rays and telluric lines}

The spikes due cosmics and defective pixels and the telluric lines have been masked following the procedure developed by Katz et al. (1998).

\subsection{Resampling to $1 D$ spectra}

The orders are normalized to their pseudo-continuum and resampled to a $1 \mathrm{D}$ spectrum with a wavelength step of $0.005 \mathrm{~nm}$ using spline interpolation (the observations are over-sampled by about $20 \%$ ).

To produce a version of the archive at the resolution 10000 (i.e. $30 \mathrm{~km} \mathrm{~s}^{-1}$ at $\lambda=550 \mathrm{~nm}$ ) the spectra are convolved by a Gaussian function of $F W H M=0.054 \mathrm{~nm}$, and rebinned with a step of $0.02 \mathrm{~nm}$.

The high resolution version has a constant resolution of $R=42000$, i.e. the resolution in $\mathrm{nm}$ changes with the wavelength. At variance, with the low resolution archive, the wavelength resolution, in $\mathrm{nm}$, is constant throughout the spectra.

Note also that the convolution to produce the low resolution archive is done on the continuum-normalized spectra, which is not formally equivalent to convolving the flux-calibrated spectra. However, the induced difference is absolutely insignificant.

\section{Flux calibration}

The goal of the flux calibration is to determine the spectral energy distribution above the atmosphere (the physi- cal flux). The standard procedure to achieve the objective (Bessell 1999) consists of determining the instrumental response and correcting for the atmospheric absorption by comparing the spectra to templates of known spectral energy distribution observed with the same setup between the target stars.

Since the primary scope of the observation was not spectrophotometry, no particular care was taken in interleaving template stars and some observations were done through heavy atmospheric absorption. Therefore, the standard procedure cannot be straightforwardly applied.

The relation between the instrumental flux deduced in the previous section and the physical flux (the flux calibration relation) primarily reflects the effects of (1) the instrumental response (i.e. the combined effect of the whole optics and CCD spectral sensitivity), (2) the absorption by a clear atmosphere and (3) an additional extinction due to an atmospheric haze.

We started from the assumptions that (1) the instrumental response is stable or at least stable during each observing run, (2) the absorption by the clear atmosphere can be parameterized by the airmass and (3) the effect of the haze can be parameterized by a color excess defined as the difference between the measured $B-V$ color and the Tycho-2 $B-V$ color.

A fit of these three functions of the wavelength using spectra of stars with known spectral energy distribution (discussed below) resulted in a photometric precision of about $5 \%$ when we considered the instrumental response stable over all observation runs.

The shape of the three functions entering the calibration relation, and the analysis of the residuals, lead to the suspicion that the correction of the atmospheric refraction provided by the spectrograph was not fully effective. This was diagnosed by (1) a stronger than expected wavelength dependence of the apparent atmospheric and haze transmissions and (2) a residual random "curvature" of the calibrated spectra that increases with airmass. Our interpretation is that the "clear atmosphere" function corrects to a first order the effect of the refraction (the star is centered on the fiber aperture by the auto-guider on the blue end of the atmospheric spectrum). The uncertainty on the centering of the star (mis-guiding) results in a "color excess" absorbed by the haze function, but depending on the value of the off-centering and of the seeing, a residual curvature remains.

It was unfortunately not possible to parameterize this effect since it depends on a random and unknown misscentering and on the seeing which is not always recorded in the observing logs. An alternative would have been to use an additional color excess to constrain the curvature, but no such independent measurement is available for the stars of our archive.

Adding a quadratic airmass term, another function of color excess and allowing for variation of the instrumental response between the different observing runs led to a photometric quality of about $3 \%$ which was then improved to $2 \%$ after corrections based on internal comparisons. 
We did not find hints for other effect on the photometric quality, such as the effect of the stress on the optical fiber or positioning of the color correction filter that may change the instrumental response.

Finally, calibration consisted in: (1) primary calibration based on the comparison with low spectral resolution flux templates (2) secondary calibration based on internal comparison and (3) correction of the instrumental response using template spectra with a better spectral resolution than for the primary calibration.

\subsection{Primary calibration}

As already explained, the observations were not originally destined for photometry and no flux templates were explicitly observed. However, since the archive contains bright stars, many of them belong also to other libraries or datasets.

The largest intersection is with the collection of low resolution spectra presented in Burnashev (1985, CDS III/126). The spectral resolution is about $R=100$ ( $F W H M=5-6 \mathrm{~nm}$ ), and the observations are from different sources which were homogenized to a common scale. The observations were done with photo-electric spectrophotometers mostly at the Crimean Observatory, and are not corrected for interstellar extinction.

Some stars in our archive are in common with other datasets with a better spectral resolution, but not in a number sufficient to fit the calibration relation. For this reason we chose to first calibrate the archive with this dataset. It was entered in the Hypercat Fits Archive (HFA) with the identification L1985BURN.

The first step was to assess the photometric quality of these templates. The $B$ and $V$ magnitudes were integrated on the spectra and compared with the measurements converted from the Tycho-2 catalogue. We found a rms difference on $B-V$ of 0.05 . The difference with the Tycho- 2 color is used to assign a weight to each of these template observations.

The determination of the calibration relation is done after the instrumental spectra from our archive are convolved to the resolution of L1985BURN. The original reference does not precisely document this resolution which varies with the origin of the observations, and we had to determine it. A first guess was obtained by fitting Gaussians on strong Balmer lines and this was fine-tuned afterwards to minimize the residuals on the strong lines. It was necessary to adopt slightly different resolutions for the blue and red parts of the spectra.

The model adopted for the primary calibration relation is:

$$
\begin{aligned}
\log \left(F_{\text {phys }}(\lambda)\right)= & \log \left(F_{\text {inst }}(\lambda)\right)+\log \left(F_{\text {inst }}^{\prime}(\lambda, \text { run })\right) \\
& +a(\lambda) \operatorname{Airm}+b(\lambda) \operatorname{Airm}^{2} \\
& +c(\lambda) E_{B-V}+d(\lambda) E_{B-V}^{\prime} .
\end{aligned}
$$

$F_{\text {phys }}$ is the physical flux reduced above the atmosphere. The two right-hand terms $\log \left(F_{\text {inst }}(\lambda)\right.$ and $\log \left(F_{\text {inst }}^{\prime}(\lambda\right.$, run $\left.)\right)$ represent the instrumental response. The first is stable over all the observing runs while the second is variable. We separated the two terms because the second could not be determined for all the observing runs: when the number of calibrated observations was less than 10 the second term was not computed and assumed to be 0 . The run-dependent instrumental response was determined for 9 runs out of 21 , and a secondary calibration (next sub-section) was also obtained.

The next two terms depend on the airmass (Airm). The first accounts for the clear atmosphere absorption and mean aperture effect due to the atmospheric extinction. The second partly corrects the "curvature" of the spectrum due to the refraction (the length of the atmospheric spectra is proportional to $\tan z, z$ being the zenithal distance).

The two last terms are parameterized by a $B-V$ color excess, defined as the difference between $B-V$ determined on our spectra and the Tycho- $2 B-V$.

It is not possible to measure directly the $B$ magnitude on our spectra since the standard $B$ band extends farther blue-ward than their limit. Hence it was necessary to "calibrate" a different color, by fitting a color equation as is usually done to convert between photometric systems. This procedure has the disadvantage that it formally depends on the actual spectral energy distribution of the star and hence may introduce a bias linked to the atmospheric parameters of the stars. However, such an effect may be estimated from the residuals of the fit of the color equation, and for small color terms (i.e. if our internal $B$ is close to the Johnson $B$ ) it can be neglected.

Since this conversion was necessary, we used this opportunity to define $2 B$ bands ( $b_{1}$ and $b_{2}$, converted to Johnson scale as $B_{1}$ and $B_{2}$ ) giving two estimates of the color excess. The difference between these two estimates gives an approximate measure of the residual curvature of the spectra due to the atmospheric dispersion.

$$
\begin{aligned}
E(B-V) & =(B-V)_{\text {Tycho }}-\left(B_{1}-V\right)_{\text {Elodie }} \\
E(B-V)^{\prime} & =B_{2}-B_{1} .
\end{aligned}
$$

The $B_{1}$ and $B_{2}$ bands were respectively defined as the integral of the flux distribution between $\lambda \lambda=450-470 \mathrm{~nm}$ and $\lambda \lambda=437-450 \mathrm{~nm}$.

The color equation was determined with the 2238 spectra of L1985BURN, using ordinary least squares and iterative rejection of outliers, as:

$b_{1}=14.830+1.001 B_{1}-0.337(B-V)$
$b_{2}=15.195+1.003 B_{2}-0.081(B-V)$.

The rms residuals from these fits are about 0.025 mag. The magnitudes and colors are practically measured on the instrumental spectra (not physical flux), and hence the measured excess is biased due to the instrumental response and depends on the airmass. Though it does not change the fit to Eq. (1), in order to have independent functions we corrected the excess from the effect of instrumental 
response and atmospheric extinction using an a posteriori fit between corrected and un-corrected excess.

The flux calibration relation was fitted on 369 spectra of stars in common between our archive and L1985BURN. The different functions of the wavelength were finally smoothed by fitting polynomials in order to erase the residual effects of resolution mis-matching which results in spurious irregularities in these functions (in particular around $\mathrm{H}_{\gamma}$ and $G$-band).

\subsection{Secondary calibration}

The primary calibration allowed us to determine the rundependent response for only half the runs (but more than half the spectra since the number of stars is not equal in each run).

The inter-comparison of spectra of the same stars for which several observations were available allowed a secondary calibration. The runs where the run-dependent response was available were used as "template".

This exercise allowed us to detect a significant change in the response of the instrument between the observations prior to 1995 and those done after. This change seems to be due to a modification of the differential refraction corrector at the end of year 1994. In addition, for two other runs the number of inter-comparisons was large enough to adopt a reliable run-dependent correction. The run-dependent corrections account for a rms photometric effect of about 0.025 mag. For 9 runs no such correction is available at present.

\subsection{Correction of the instrumental response}

Up to this point the flux calibration can be assumed to properly correct the effect of atmospheric absorption and to correct at best the aperture effect due to the differential refraction. However, the instrumental response was evaluated using low-resolution spectra and furthermore had to be smoothed. At variance with the other functions which presumably vary smoothly with wavelength, the instrumental response may be more chaotic.

Therefore, the last step of the calibration process consists of using higher spectral resolution $(R \approx 1000)$ to correct the instrumental response. These reference spectra are taken from the following dataset, referenced by their HFA identification:

- L96111KP1 and L96111KP2. The ELODIE database contains 191 spectra of 144 stars from the Jones library (Leitherer et al. 1998) $(R=2800)$. This library cover two narrow wavelength ranges, one in the blue part below $\lambda=451 \mathrm{~nm}$ the other between $\lambda \lambda=478-547 \mathrm{~nm}$. This is the highest resolution reference for stellar spectra yet available for comparison. This library is not corrected for the aperture effect due to differential refraction and is not of photometric quality. After recalibrating the "color" of these spectra (defining special color-band as we did for our spectra for the pri- mary calibration), the mean agreement with our spectra is satisfactory;

- L1996SERO. The ELODIE database has 8 spectra of 6 stars in common with Serote Ross et al. (1996). These spectra are from CFH $(R=650)$ and OHP $(R=4000)$ observations. They intersect the red half of our spectral range. The comparison with the L1996SERO spectra, after the spectral resolutions are matched by convolving our spectra with a Gaussian, show pixel-to-pixel variations of the order of $2 \%(\mathrm{rms})$ and low-frequency variations of typically $5 \%$. These figures are consistent with the comparison performed by Serote Ross et al. (1996) with the Silva \& Cornell (1992) library;

- L1984JACO. We have 2 spectra for 2 stars (HD 094028 \& HD 028099) in common with the Jacoby library ( $R=1200$ ) which cover the full wavelength range. The two spectra compared with L1984JACO reveal a significant gradient (the difference over the whole range of wavelength are respectively $29 \%$ and $14 \%$ for the first and second spectrum) and a pixel-to-pixel variation of $0.5 \% \mathrm{rms}$. The $B-V$ color integrated from the L1984JACO spectra disagrees with the Tycho-2 value in amounts that explain the differences with our spectra;

- L1987KIEH. We have 13 spectra of 7 stars from the dataset of Kiehling (1987) $(R=800)$;

- L1994DANK. We have 17 spectra from 14 stars in common with Danks \& Dennefeld (1994) $(R=1500)$. The red half of our wavelength range intersects with L1994DANK;

- L1983GUNN. The Gunn \& Stryker (1983) library $(R=500)$ counts 5 stars in common with our archive;

- L1984NSOA. We compared our 7 solar spectra with the Solar flux atlas (Kurucz et al. 1984) $(R=500000$, also stored in HFA with $R=10000)$.

We made all the individual comparisons between our spectra, convolved by a Gaussian to match the resolution, and these references. Because the broad-band variations are probably due either to random photometric errors in our archive (certainly not systematic), correction of the interstellar extinction (it was not always possible to de-correct the templates) or errors in the templates, we subtracted a low-degree polynomial ( 1 to 3 depending on the dataset) to these comparisons.

The individual comparisons (see Fig. 3) were then combined and produced a mean residual convolved to a resolution of $2 \mathrm{~nm} F W H M$ (each order of the original spectrum covers about $4 \mathrm{~nm}$ and no correction of the instrumental response at a lower scale is desired). This was used to correct the instrumental response.

We also made an unsuccessful attempt to compare our archive with the Pickles (1998) library (L1998PICK): all the spectra were compared to the nearest (in the $T_{\text {eff }}-\log g-[\mathrm{Fe} / \mathrm{H}]$ space) template in L1998PICK. But the mapping of the parameters space in L1998PICK is not dense enough and the residuals are too affected by the distance in the parameter space to permit a useful analysis. 


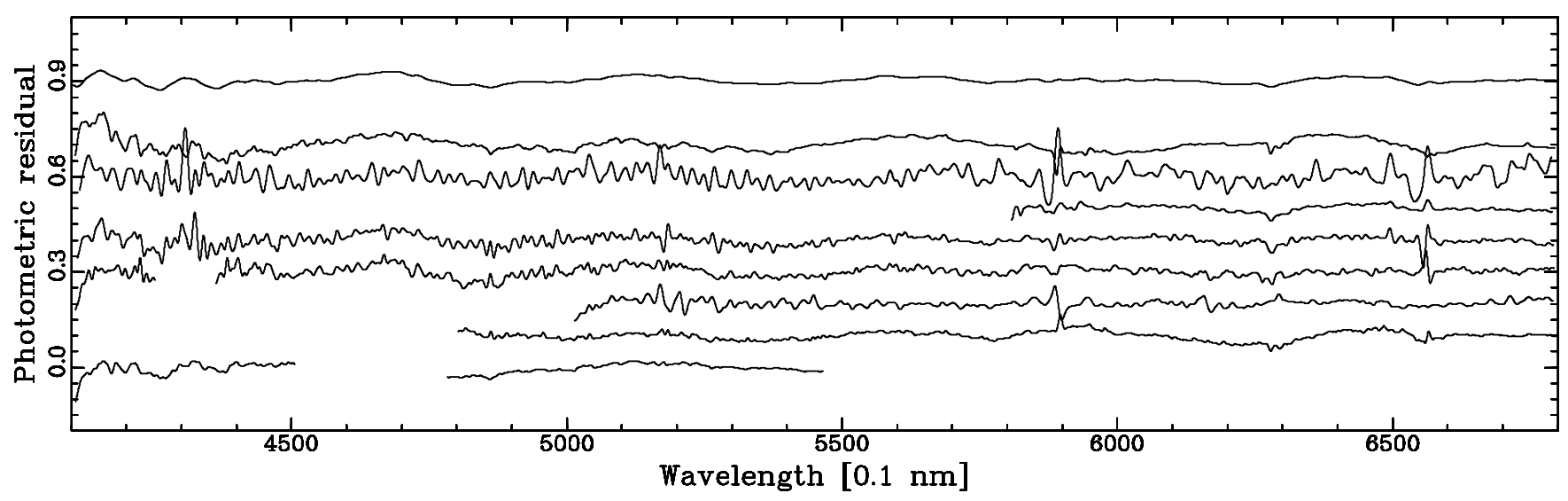

Fig. 3. The mean photometric residuals of the 9 libraries used for the correction of the instrumental response (before this corrections). The ordinate is the mean difference (Elodie - reference library) in units of relative physical flux (normalized to 1 at $555 \mathrm{~nm}$ ). Each dataset is shown on a different line, each being arbitrary $y$-shifted by 0.05 . From bottom to top they are: Jones (blue and red), Serote Roos et al. (OHP and CFH), Jacoby, Kiehling, Danks \& Dennefeld, Gunn \& Stryker and the Sun. For the purpose of the display these spectra have been slightly smoothed. The top line is the combination of all the individual comparisons which were used to compute the correction to the instrumental response

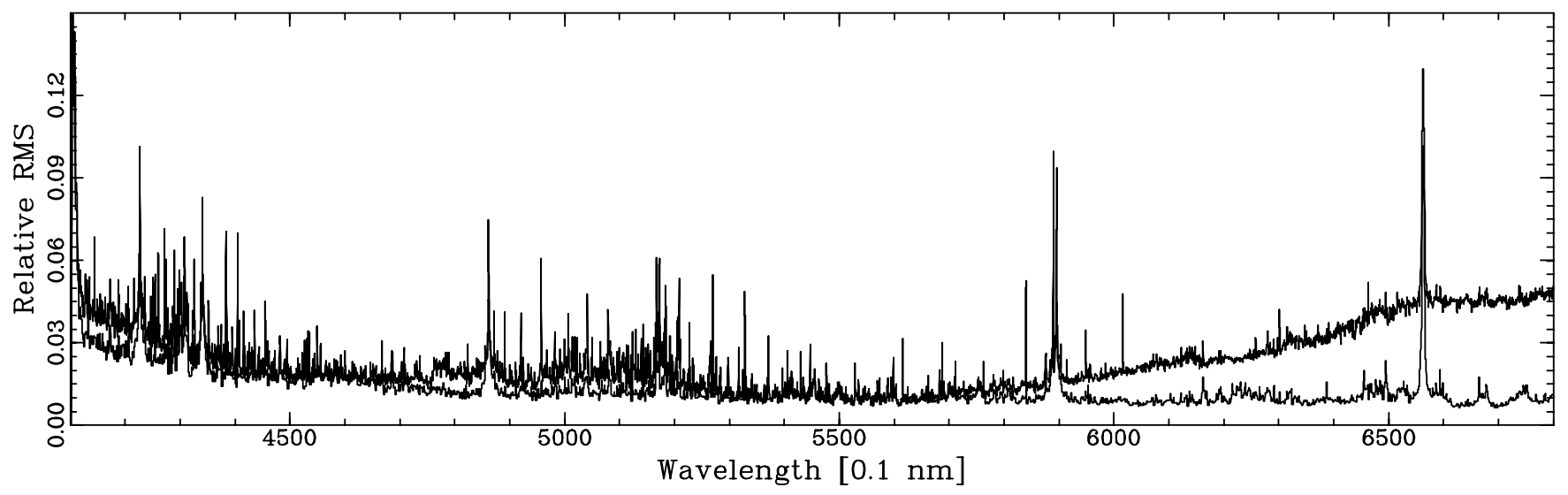

Fig. 4. Photometric precision deduced from the comparison of multiple observations of the same star. The ordinate are the rms of the 358 pair comparisons $\left(\ln \left(\right.\right.$ Flux $_{2} /$ Flux $\left._{1}\right)$ ) (the spiky appearance is due to the logarithmic comparison). The higher curve is the total $\mathrm{rms}$ while the lower one is after third degree polynomials were subtracted from each individual comparison: this disentangles the effect of photon noise and uncertainty on the long range flux calibration (due to the differential refraction). To obtain the order of magnitude of the photometric error on single observation, the ordinates should be divided by $\sqrt{2}$

We did not attempt to interpolate the spectra within the Pickles library.

\section{Quality control}

To check the reliability of our data and reduction we used the following tests: (1) analyze the inter-comparison of repeated observations and analyze internal consistency, (2) compare fully reduced spectra with other libraries and (3) compare Lick indices for stars with published measurements.

We investigated three possible sources of discrepancy: (1) errors on the instrumental response and/or correction of atmospheric extinction are likely to result in low and intermediate spatial frequencies variations. (2) inaccurate subtraction of the diffuse light may be diagnosed as significant mismatch of the strongest lines and (3) inaccurate correction for the blaze will lead to oscillations with a oneorder period.

\subsection{Internal consistency}

\subsubsection{Multiple observations of the same star}

There are in total 358 repeated observations for 104 stars in the archive. After giving a double weight to the observations in the runs for which the run-dependent response was computed from primary calibration and half weight to the observations done with an airmass larger than 1.5 (their photometric quality is lower because of the differential refraction), we find a global rms dispersion for the pairwise comparison of about $3.5 \%$, see Fig. 4 . Without weighting, the dispersion is $4 \%$ and the difference was a hint of the lowest photometric quality of high airmass observations, 
hence leading to suspicion about the refraction corrector. Since it is a pairwise comparison, the mean photometric error on individual observations is: $3.5 / \sqrt{2}=2.5 \%$. (Note that this error does not account for possible systematic errors affecting uniformly the whole archive, this will be discussed in Sects. 5.2 and 5.3).

Most of the photometric errors lie in the residual "curvature" of the spectra. After subtracting a third degree polynomial from each pairwise comparison, the rms dispersion becomes $0.7 \%$, corresponding to a mean photometric precision of $0.5 \%$, in full agreement with the mean $S / N$ ratio. No hint for a modulation at the scale of one order of the echelle spectra is found.

\subsubsection{Modeled spectra}

Random errors on the strengthening of the spectra are probably on average smaller than $0.5 \%$ because otherwise they would increase the dispersion of the pairwise internal comparison (after the third degree polynomial is subtracted).

The new version of the TGMET program that will be described in a separate paper allowed us to generate interpolated spectra for a given position in the $\left(T_{\text {eff }}, \log g,[\mathrm{Fe} / \mathrm{H}]\right)$ space. The difference between the spectra in the archive and these modeled spectra can potentially uncover possible random errors on the strengthening.

Therefore we computed and averaged the differences to the modeled spectra for all the spectra in the archive. The mean residuals were searched for a modulation at the scale of one order, either in the spectrum or in its Fourier transform. We did not detect any signal. We made simulations that suggested that a $0.5 \%$ (rms) modulation would probably have been detected, but a $0.2 \%$ modulation would not.

\subsection{External comparisons}

The comparisons between our physically calibrated spectra and other libraries were used in the previous section to compute the correction to the instrumental response. The goal was to determine the variation of the response at the scale of 1 to $5 \mathrm{~nm}$ that was not constrained by the primary calibration. Therefore, by construction, the mean external comparison is flat (i.e. may only vary smoothly with the wavelength). A careful analysis of the individual comparison could assess the level of stability of the instrumental response.

The patterns in the correction to the instrumental response were consistently found in the different individual comparisons and no significant effect was found after this correction was applied. However, the scarcity of the available comparisons did not allow us to measure a photometric precision.

\subsection{Comparison of Lick indices}

The Lick indices (see e.g. WO97) measure equivalent widths of features defined in bands of 2 to $5 \mathrm{~nm}$ (hence of the width of about $1 / 2$ or 1 order of the echelle spectra). Their measurements are then sensitive to both incorrect strengthening of the spectra and errors in the instrumental response.

The spectral range of the Elodie archive allowed us to measure most of the Lick indices. Only the blue $\mathrm{CN}$ indices are missed as well as the $\mathrm{H} \delta$ indices $\left(\mathrm{H} \delta_{\mathrm{A}}\right.$ and $\left.\mathrm{H} \delta_{\mathrm{F}}\right)$ defined in WO97.

We used the definitions from WO97 or Cardiel et al. (1998) to measure the indices for all the spectra in the archive. The results are reported in Table 1 (given in the electronic version only).

The measurement errors computed from the photon noise (see e.g. Prugniel et al. 2001) is in the present case negligible because of the extremely high $S / N$ per $\AA$. The main source of error is due to the photometric calibration and may be systematic. The comparison of the Lick indices measured in WO97 constrain the magnitude of these systematic errors.

The archive has 102 stars which have been previously measured in WO97. The comparisons are reported in Table 2.

Table 2. Comparison of Lick indices: Col. 1: Name of the Lick index Col. 2: Number of spectra used for the comparison Col. 3: Mean value of the index Col. 4: Mean shift (Elodie) - (WO97) Col. 5: rms dispersion of the comparison Col. 6: Majorant of photometric error

\begin{tabular}{|c|c|c|c|c|c|}
\hline Index & $\#$ & Mean & Shift & $\mathrm{rms}$ & Error \\
\hline $\mathrm{Ca} 4227$ & 101 & 0.689 & -0.091 & 0.278 & -0.0073 \\
\hline G4300 & 102 & 3.858 & 0.083 & 0.464 & 0.0024 \\
\hline $\mathrm{H} \gamma_{\mathrm{A}}$ & 102 & -1.765 & 0.392 & 0.527 & 0.0089 \\
\hline $\mathrm{H} \gamma_{\mathrm{F}}$ & 99 & 0.678 & 0.177 & 0.337 & 0.0084 \\
\hline $\mathrm{Fe} 4383$ & 102 & 2.715 & -0.341 & 0.692 & -0.0067 \\
\hline Ca4455 & 101 & 0.723 & -0.267 & 0.250 & -0.0118 \\
\hline Fe4531 & 101 & 2.299 & -0.078 & 0.335 & -0.0017 \\
\hline $\mathrm{Fe} 4668$ & 102 & 2.214 & -0.183 & 0.712 & -0.0021 \\
\hline $\mathrm{H}_{\beta}$ & 103 & 2.706 & 0.061 & 0.192 & 0.0021 \\
\hline Fe5015 & 102 & 3.393 & -0.197 & 0.449 & -0.0026 \\
\hline $\mathrm{Mg}_{1}$ & 103 & 0.040 & 0.003 & 0.011 & 0.0030 \\
\hline $\mathrm{Mg}_{2}$ & 103 & 0.125 & -0.005 & 0.011 & -0.0054 \\
\hline $\mathrm{Mg}_{b}$ & 100 & 2.335 & 0.117 & 0.247 & 0.0036 \\
\hline $\mathrm{Fe} 5270$ & 100 & 1.777 & -0.088 & 0.254 & -0.0022 \\
\hline Fe5335 & 102 & 1.515 & -0.035 & 0.279 & -0.0009 \\
\hline Fe5406 & 103 & 0.895 & 0.076 & 0.279 & 0.0028 \\
\hline Fe5709 & 101 & 0.505 & -0.046 & 0.187 & -0.0020 \\
\hline Fe5782 & 101 & 0.313 & -0.095 & 0.168 & -0.0047 \\
\hline $\mathrm{NaD}$ & 103 & 1.415 & -0.165 & 0.408 & -0.0051 \\
\hline $\mathrm{TiO}_{1}$ & 103 & 0.006 & -0.006 & 0.011 & -0.0057 \\
\hline $\mathrm{TiO}_{2}$ & 103 & 0.011 & 0.005 & 0.009 & 0.0054 \\
\hline
\end{tabular}




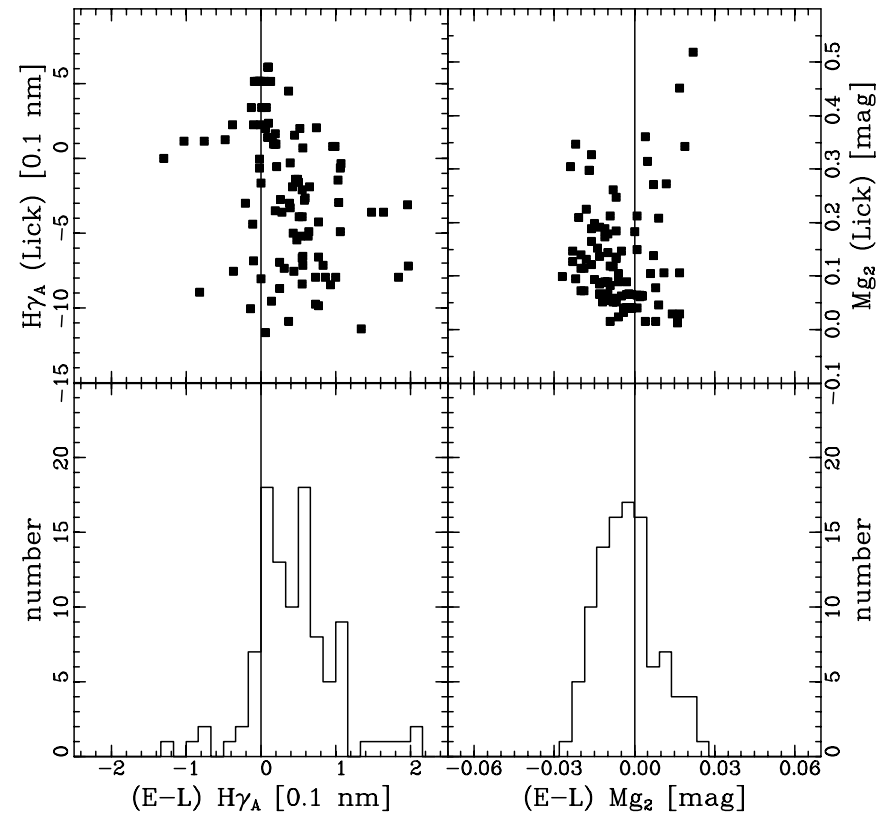

Fig. 5. Comparison of Lick indices $\mathrm{Mg}_{2}$ and $\mathrm{H} \gamma_{\mathrm{A}}$ with WO97. The abcissae are the difference (Elodie - WO97) between the Lick indices. The top panels show the distribution as a function of the value in WO97, and the bottom panels the histograms of the differences

We do not find systematic effects that would result from errors in the instrumental errors and the residual dispersions are compatible with the errors in the WO97 measurements (the internal error due to the photon noise in the archive is totally negligible).

The systematic shifts are always smaller than the dispersion except for Ca4455 (where it is equal) and for Fe4383 the shift may be significant. However, we cannot attribute this to an error in our measurements and in particular we note that the shift for Fe4383 is similar to the one found by WO97 when they compared the original Lick measurements with the spectra of the Jones library (note that our flux calibration is not fully independent from the Jones library since this latter was used among others to correct the instrumental response). The Ca4455 index is not in the range of the Jones spectra and we cannot comment of the shift we found. Figure 5 presents the distributions of the differences (us - WO97) for two indices: $\mathrm{Mg}_{2}$ and $\mathrm{H} \gamma_{\mathrm{A}}$. The first one is very important for extragalactic studies, it is defined on a broad region ( $53 \mathrm{~nm}$ between the extrema of the red and blue side-bands). Though it is potentially sensitive to the uncertainties on the flux calibration described above, the agreement between us and WO97 is excellent. The second index presented in Fig. 5, $\mathrm{H} \gamma_{\mathrm{A}}$, is narrower (14 nm) but it is located in the blue region of our spectra where our calibration is probably less accurate. There is a systematic shift with respect to WO97, but the rms residual is fully accounted for by the errors on WO97.

\section{Data format and public access}

The spectra are stored in HFA in the form of FITS files containing 4 extensions (Grøsbol et al. 1988):

The first extension is a $1 \mathrm{D}$ image header data unit (HDU) containing the spectra calibrated in wavelength expressed in the rest-frame of each star and sampled between 410 and $680 \mathrm{~nm}$. The high resolution version is normalized with a step between the sampling points of $0.005 \mathrm{~nm}$ and the low resolution version is given in physical flux with a step of $0.020 \mathrm{~nm}$.

Another image HDU (EXTNAME=NOISE), with the same sampling, contains the noise attached to the values in the primary HDU (in the unit of this spectra). Pixels are independent.

The other extensions are the flux calibration relations which enable to convert the instrumental flux into physical or normalized flux. They are binary table extensions and the relations are coded as spline representations. The details are given in the Pleinpot user's manual ${ }^{3}$, and the conversions can be done on-the-fly with the pipeline attached to HFA.

The physical flux calibration $($ EXTNAME $=$ FCAPHY $)$ and the normalized flux relation $($ EXTNAME $=$ FCANOR) have a spectral resolution of about $2 \mathrm{~nm}$.

Each header, which includes the keywords created during the observations and TACOS reduction, have been completed with useful information on the star: spectral type, Tycho- $2 V$ and $B-V$, absolute magnitude $M_{V}$ and $\left(T_{\text {eff }}, \log g,[\mathrm{Fe} / \mathrm{H}]\right)$ with their reliability flag, and $v_{\mathrm{r}}$. The nominal $F W H M$ of the spectrum can also be retrieved in the header.

In addition to the spectra of the stars, the archive contains the different intermediate files used along the data processing. They are described in the electronic version of the paper.

\section{Conclusion}

We have presented an archive of 908 spectra of 709 stars at two resolutions, $R=10000$ and $R=42000$, and in the wavelength range $\lambda \lambda=410-680 \mathrm{~nm}$. The high resolution version is archived normalized to its pseudo-continuum while the low resolution is given in physical flux (above the earth atmosphere) normalized to one at $\lambda=555 \mathrm{~nm}$.

The mean $S / N$ per pixel in the high resolution version of the archive is $150(S / N \approx 700$ per $0.1 \mathrm{~nm})$, the photometric precision of the strengthening of the orders of the echelle spectra is better than $0.5 \%$, the precision in bands of about $10-20 \mathrm{~nm}$ is $0.5 \%$ and the overall (broadband) precision is $2.5 \%$, limited by the effect of differential refraction.

The stars in this archive cover a large range of the parameter space, though the mapping is still crude for hot and very cool stars.

\footnotetext{
3 http://www-obs. univ-lyon1.fr/ prugniel/cgi-bin/ pleinpot/pleinpot.html
} 
This archive supersedes the TGMET (1996) library and is being used for automatic determination of the stellar parameters $\left(T_{\text {eff }}, \log g,[\mathrm{Fe} / \mathrm{H}]\right)$ by direct comparison of spectra with interpolations in the archive. The new version of the TGMET program, still under development, will enable determinations of the parameters from any $R>10000$ spectra, independently of the detailed observational setup. It will be presented in a future paper.

The archive will also be used to synthesize extragalactic stellar populations and to use these high resolution synthetic spectra as kinematical templates to determine simultaneously the parameters of the stellar population and the line-of-sight velocity distribution.

The archive itself will be completed with new observations and spectra from the OHP archive with the goal of improving the mapping of the parameter space and the quality of the photometric calibration.

Acknowledgements. We thank the director and technical staff of Observatoire de Haute-Provence for their constant support of our observing programs and for providing some spectra retrieved from the ELODIE archive. We thank all the observers who provided their observations to complete the database, especially the COROT team: D. Ballereau, J.-C. Bouret, C. Catala, T. Hua, D. Katz, F. Lignieres, T. Lueftinger and C. Van't Veer. We acknowlegde, with gratitude, financial support from the GDR Galaxies, CNRS, France. This work made extensive use of the SIMBAD and VIZIER databases, operated at CDS, Strasbourg, France.

\section{References}

Alonso, A., Arribas, S., \& Martínez-Roger, C. 1996a, A\&A, 313,873

Alonso, A., Arribas, S., \& Martínez-Roger, C. 1996b, A\&AS, 117,227

Alonso, A., Arribas, S., \& Martínez-Roger, C. 1999a, A\&AS, 139,335

Alonso, A., Arribas, S., \& Martínez-Roger, C. 1999b, A\&AS, 140,261
Baranne, A., Queloz, D., Mayor, M., et al. 1996, A\&AS, 119, 373

Bessell, M. S. 1999, PASP, 111, 1426.

Burnashev, V. I. 1985, Abastumanskaya Astrofiz. Obs., Byull., 59, 83 (CDS III/126)

Blackwell, D. E., \& Lynas-Gray, A. E. 1998, A\&AS, 129, 505

Cardiel, N., Gorgas, J., Cenarro, J., \& Gonzalez, J. 1998, A\&AS, 127, 597

Carney, B. W., Latham, D. W., Laird, J. B., \& Aguilar, L. A. 1994, AJ, 107, 2240.

Cayrel de Strobel, G., Soubiran, C., Friel, E. D., Ralite, N., \& François, P. 1997, A\&AS, 124, 299

Cayrel de Strobel, G., Soubiran, C., \& Ralite, N. 2001, A\&A, submitted

Danks, A. C., \& Dennefeld, M. 1994, PASP, 106, 382

Donati, J.-F., Semel, M., Carter, B. D., Rees, D. E., \& Cameron, A. C. 1997, MNRAS, 291, 658

Fioc, M., \& Rocca-Volmerange, B. 1997, A\&A, 326, 950

Grøsbol, P., Harten, R. H., Greisen, E. W., \& Wells, D. C. 1988, A\&AS, 73, 359

Gunn, J. E., \& Stryker, L. L. 1983, ApJS, 52, 121

Høg, E., Fabricius, C., Makarov, V. V., et al. 2000, A\&A, 355, 27

Jacoby, G. H., Hunter, D. A., \& Christian, C. A. 1984, ApJS, 56,257

Katz, D., Soubiran, C., Cayrel, R., Adda, M., \& Cautain, R. 1998, A\&A, 338, 151

Kiehling, R. 1987, A\&AS, 69, 465

Kurucz, R. L., Furenlid, I., Brault, J., \& Testerman, L. 1984, (ftp://ftp.noao.edu/fts/fluxatl/)

Pickles, A. J. 1998, PASP, 110, 863

Prugniel, Ph., Maubon, G., \& Simien, F. 2001, A\&A, in press Queloz, D. 1996, ELODIE user's guide (http://www.obs-hp.fr/)

Serote Roos, M., Boisson, C., \& Joly, M. 1996, A\&AS, 117, 93

Silva, D. R., \& Cornell, M. E. 1992, ApJS, 81, 865

Soubiran, C., Katz, D., \& Cayrel, R. 1998, A\&AS, 133, 221

Thévenin, F. 1998, BICDS, 49

Vazdekis, A. 1999, ApJ, 513, 224

Worthey, G., \& Ottaviani, D. L. 1997, ApJS, 111, 377 (http://astro.sau.edu/ worthey/html/system.html) (WO97) 